\title{
USO DE MEMBRANAS CERÂMICAS MICROPOROSAS NA PRODUÇÃO DE EMULSÕES ÓLEO DE GIRASSOL EM ÁGUA
}

\author{
V. ZANATTA ${ }^{1 *}$, K. REZZADORI, F. M. PENHA ${ }^{1}$, G. ZIN ${ }^{1}$, J. C. C PETRUS ${ }^{1}$, M. DI LUCCIO ${ }^{1}$ \\ ${ }^{1}$ Universidade Federal de Santa Catarina, Departamento de Engenharia Química e Engenharia de \\ Alimentos \\ E-mail para contato: zanattavane@gmail.com
}

\begin{abstract}
RESUMO - Emulsificação com membrana é um método simples quando comparado aos métodos convencionais e tem recebido atenção crescente ao longo dos últimos anos, com aplicações potenciais em muitas áreas. Neste estudo foi avaliado o preparo de emulsões óleo/água $(\mathrm{O} / \mathrm{A})$ em sistema de microfiltração tangencial utilizando membranas cerâmicas com tamanho de poros 0,2 e $0,8 \mu \mathrm{m}$, óleo de girassol como fase dispersa e soluções de água e polissorbato 80 como fase contínua. O tamanho, a distribuição de tamanho de gotas e a estabilidade das emulsões foram avaliados em função da concentração de óleo (10\% a 20\%), concentração de surfactante $(1 \%$ a $4 \%)$, a velocidade tangencial $\left(0,12 \mathrm{~m} \cdot \mathrm{s}^{-1}\right.$ a $\left.0,24 \mathrm{~m} \cdot \mathrm{s}^{-1}\right)$ e a pressão de operação ( 1 bar a 3 bar). O processo de emulsificação com membrana permitiu se obter emulsões com $\mathrm{D}_{3,2}$ variando de 2,9 a $16 \mu \mathrm{m}$ e amplitude das distribuições (span) entre 1,5 e 7,0. Os resultados podem ser explicados por uma influência direta do tamanho dos poros da membrana e pelos parâmetros estudados, sendo que, todos mostraram ter influências significativas no processo de emulsificação. As emulsões produzidas com a membrana de 0,2 $\mu \mathrm{m}$ apresentaram a maior estabilidade e $\mathrm{D}_{3,2}$ e spans menores que as emulsões obtidas pelo método convencional utilizando rotor-estator.
\end{abstract}

\section{INTRODUÇÃO}

Emulsões são comumente utilizadas em diversas indústrias, como a de cosméticos, farmacêutica, alimentícia e de tintas. De interesse para a tecnologia de alimentos podem-se considerar as emulsões de óleo em água $(\mathrm{O} / \mathrm{A})$ e água em óleo $(\mathrm{A} / \mathrm{O})$. As primeiras, de maior interesse, são constituídas de gotas de óleo suspensas em uma fase aquosa contínua, enquanto as emulsões $\mathrm{A} / \mathrm{O}$, são constituídas de gotas de água suspensas em uma fase oleosa contínua (NAZIR, 2010). Emulsões de óleo em água (O/A) são as mais versáteis, e as que levam a um maior número de aplicações, sendo produzidas pela homogeneização das fases dispersa e aquosa na presença de um ou mais emulsificantes. 
A estabilidade das emulsões formuladas é importante, uma vez que, quanto mais estável é a emulsão, maior a aplicabilidade e vida de prateleira do produto que a utiliza. A estabilidade da emulsão está intimamente relacionada com a distribuição do tamanho de gota, uma vez que as distribuições mais largas de tamanho da gota podem aumentar o efeito de amadurecimento de Ostwald, com o aumento do tamanho das gotas maiores, que por sua vez, favorece a coalescência e a separação de fases. A estabilidade necessária para emulsão depende da aplicação desta emulsão (MATOS, 2013).

Nos últimos anos, a emulsificação com membranas (EM) tem recebido um crescente destaque por tratar-se de um processo simples e com aplicações potenciais em diversas áreas. O método utilizado para a preparação de emulsões tem uma grande influência sobre as propriedades físicoquímicas do produto final. O tamanho da gota e a distribuição de tamanho, estão entre as mais importantes propriedades que devem ser consideradas ao preparar um determinado tipo de emulsão. É justamente nesta etapa que as membranas podem ser úteis, pois podem produzir micropartículas com pouca dispersão quanto ao tamanho (NAKASHIMA, 1991). Neste contexto, o objetivo deste trabalho foi avaliar o preparo de emulsões de óleo de girassol em água pelo método de emulsificação com membrana cerâmica em modo tangencial, estudando-se os efeitos dos parâmetros de emulsificação (velocidade tangencial, pressão, concentração de óleo e surfactante) sobre as características das emulsões (tamanho médio e distribuição de tamanho de gotas) e estabilidade destas.

\section{MATERIAL E MÉTODOS}

\subsection{Materiais}

No preparo das emulsões utilizou-se óleo de girassol (SINHÁ) adquirido em um mercado local com uma viscosidade de $55,62 \pm 1,69 \mathrm{mPa} \cdot \mathrm{S}$ e uma densidade de $910 \mathrm{~kg} \cdot \mathrm{m}^{-3}$. A fase contínua era constituída pelo surfactante polissorbato 80 (polietilenoglicol monooleato de sorbitano), que é um surfactante não iônico, de grau alimentício, com massa molar $1310 \mathrm{~g} \mathrm{~mol}^{-1}$ e concentração micelar crítica (CMC) de $1,2 \times 10^{-5} \mathrm{~mol} \mathrm{~L}^{-1}$.

Foram utilizadas duas membranas $(0,2$ e $0,8 \mu \mathrm{m})$ cerâmicas monocanal hidrofílicas, preparadas à base de alumina (Ceraver, França) com área filtrante útil de aproximadamente $50 \mathrm{~cm}^{2}$, com diâmetro externo de $10 \mathrm{~mm}$, diâmetro interno de $8 \mathrm{~mm}$ e $250 \mathrm{~mm}$ de comprimento. Segundo o fabricante, esta membrana apresenta uma elevada dispersão de tamanhos de poros e alta porosidade da ordem de $55 \%$. 


\subsection{Procedimento Experimental}

Para a produção das emulsões utilizou-se um sistema de filtração operando em escoamento tangencial. Ao passar pelos poros da membrana, o óleo é fracionado em pequenas gotículas, resultando em uma emulsão de óleo em água. A Figura 1 apresenta um esquema do aparato experimental.

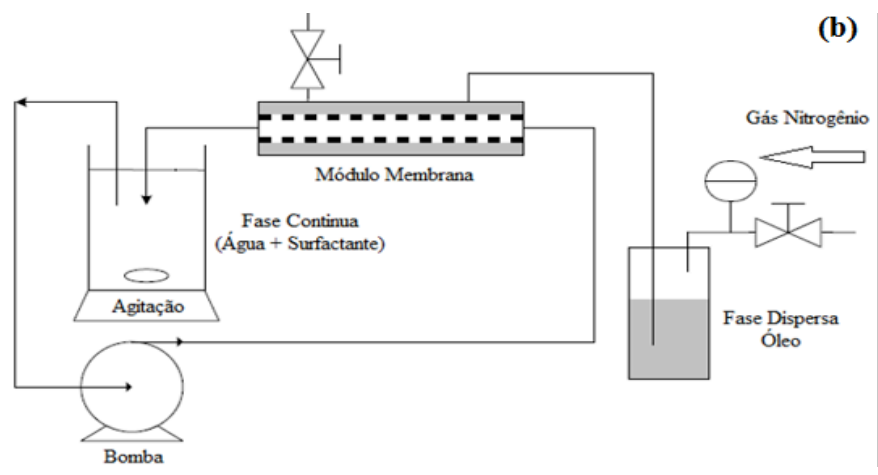

Figura 1: Diagrama esquemático do sistema experimental utilizado para emulsificação com membranas.

Para avaliação das variáveis relevantes no processo foi utilizado um planejamento experimental do tipo Plackett \& Burman, constituído por oito ensaios lineares nos níveis -1 e +1 , e três ensaios no ponto central. Foram selecionadas quatro variáveis independentes: pressão (bar), velocidade tangencial $\left(\mathrm{m} \cdot \mathrm{s}^{-1}\right)$, concentração de óleo e de surfactante $(\%)$. Como resposta foram monitorados o Span e o $\mathrm{D}_{3,2}$. Os ensaios foram realizados com ambas membranas. Salienta-se que para a membrana de $0,8 \mu \mathrm{m}$, a pressão utilizada foi menor, devido ao tamanho dos poros da membrana.

\subsection{Determinação da Distribuição do Tamanho das Gotas}

As determinações da distribuição do tamanho das gotículas foram realizadas pelo método de difração a laser (Mastersizer 2000, Malvern InstrumentsLtd, Worcestershire, Inglaterra). Os parâmetros obtidos das análises por difração a laser para caracterizar a distribuição do tamanho de gota foram o diâmetro médio de Sauter $\left(\mathrm{D}_{3,2}\right)$ e o fator Span. O diâmetro de Sauter é calculado conforme a Equação (1) (JOSCELYNE, 2000). Os dados de distribuição de tamanho podem ser usados para calcular os coeficientes de variação (Spans), conforme a Equação 2, medindo-se dessa forma, a largura da distribuição. Quanto mais estreita é a distribuição, menor será o intervalo. Se o 
coeficiente de variação (CV) é inferior a 0,4 , as gotículas podem ser consideradas monodispersas (JOSCELYNE, 2000).

$$
D_{3,2}=\frac{\sum_{i} n_{i} d_{i}^{8}}{\sum_{i} n_{i} d_{i}^{2}}
$$

sendo $\mathrm{n}_{\mathrm{i}} \mathrm{o}$ número de gotas de diâmetro di.

$$
\operatorname{Span}=\frac{d_{90}-d_{10}}{d_{50}}
$$

sendo $\mathrm{d}_{10}, \mathrm{~d}_{50}$ e $\mathrm{d}_{90}$ os diâmetros máximos que correspondem $10 \%, 50 \%$ e $90 \%$ do volume total de gotas da emulsão, ou seja, $10 \%$ das gotas da emulsão têm diâmetro menor que $\mathrm{d}_{10}$, e assim sucessivamente.

\section{RESULTADOS E DISCUSSÃO}

\subsection{Emulsões Produzidas com Membrana de 0,2 $\mu \mathrm{m}$}

A Figura 2(a) apresenta a distribuição de tamanho de gotas das emulsões obtidas com a membrana de 0,2 $\mu \mathrm{m}$, analisadas logo após o preparo. Pode-se observar que a maioria das condições experimentais resultou em emulsões com distribuição de tamanho de gotas bimodal. Por outro lado, os pontos centrais e o ensaio 8 apresentaram uma distribuição multimodal e os ensaios 1 , 2 e 3 apresentaram uma tendência ao comportamento monomodal. Foram analisadas as respostas do tamanho do Span e o diâmetro das partículas $\left(\mathrm{D}_{3,2}\right)$ no tempo zero $\left(\mathrm{t}_{0}\right)$ e, após 60 dias de armazenamento $\left(\mathrm{t}_{1}\right)$ para a membrana de $0,2 \mu \mathrm{m}$.

Após 60 dias de armazenamento, os ensaios centrais e os ensaios 6 e 8 apresentam um pequeno aumento na distribuição do tamanho das gotas. Esse comportamento é nítido e pode ser visualizado na Figura 2(b). A grande diferença entre esses ensaios, está nos parâmetros utilizados. Por exemplo, para o ensaio 8, todas as variáveis utilizadas, pressão, concentração de surfactante, concentração de óleo e velocidade tangencial, estavam nos níveis menores.

Os dados de tamanho médio de gotas e span para cada condição experimental são apresentados na Tabela 1. Observa-se que os pontos centrais e o ensaio 8 apresentam os maiores tamanhos médios de gotículas, sendo aproximadamente 3 vezes maior que os demais ensaios. Os ensaios 6 e 8 apresentam uma larga distribuição no tamanho das gotas, quando comparados aos demais. No entanto, os ensaios 1 e 5 apresentaram tamanhos de gotas menores e um estreito tamanho da distribuição das gotas. 
Tabela 1: Variação de $\mathrm{D}_{3,2}(\mu \mathrm{m})$ e span das emulsões obtidas com a membrana de $0,2 \mu \mathrm{m}$ em função da pressão, velocidade tangencial e das concentrações de surfactante e de óleo. T0 se refere a emulsões analisadas logo após o preparo, e T1 a emulsões analisadas 60 dias após o preparo

\begin{tabular}{|c|c|c|c|c|c|c|c|c|}
\hline \multirow[t]{2}{*}{ Ensaios } & \multicolumn{2}{|c|}{ Pressão Surfactante } & \multirow{2}{*}{$\begin{array}{l}\text { Óleo } \\
(\%)\end{array}$} & \multirow{2}{*}{$\begin{array}{c}\text { Velocidade } \\
\text { Tangencial }\left(\mathbf{m} \cdot \mathbf{s}^{-1}\right)\end{array}$} & \multirow{2}{*}{$\begin{array}{c}\mathrm{D}\left[{ }_{3,2}\right] \\
\mathrm{T}_{0}\end{array}$} & \multirow{2}{*}{$\begin{array}{c}\mathrm{D}\left[{ }_{3,2}\right] \\
\mathrm{T}_{1}\end{array}$} & \multirow{2}{*}{$\begin{array}{c}\text { Span } \\
\mathrm{T}_{0}\end{array}$} & \multirow{2}{*}{$\begin{array}{c}\text { Span } \\
\mathrm{T}_{1}\end{array}$} \\
\hline & (bar) & $(\%)$ & & & & & & \\
\hline 1 & $3(+1)$ & $1(-1)$ & $10(-1)$ & $0,24(+1)$ & 2,91 & 2,95 & 1,79 & 1,82 \\
\hline 2 & $3(+1)$ & $4(+1)$ & $10(-1)$ & $0,12(-1)$ & 2,94 & 3,60 & 1,92 & 2,43 \\
\hline 3 & $3(+1)$ & $4(+1)$ & $20(+1)$ & $0,12(-1)$ & 3,08 & 3,16 & 1,95 & 2,01 \\
\hline 4 & $1,5(-1)$ & $4(+1)$ & $20(+1)$ & $0,24(+1)$ & 3,25 & 3,95 & 1,86 & 2,34 \\
\hline 5 & $3(+1)$ & $1(-1)$ & $20(+1)$ & $0,24(+1)$ & 2,92 & 3,09 & 1,59 & 1,68 \\
\hline 6 & $1,5(-1)$ & $4(+1)$ & $10(-1)$ & $0,24(+1)$ & 4,04 & 4,76 & 2,52 & 2,98 \\
\hline 7 & $1,5(-1)$ & $1(-1)$ & $20(+1)$ & $0,12(-1)$ & 3,64 & 3,92 & 1,97 & 2,57 \\
\hline 8 & $1,5(-1)$ & $1(-1)$ & $10(-1)$ & $0,12(-1)$ & 11,56 & 13,84 & 2,16 & 2,96 \\
\hline 9 & $2,25(0)$ & $2,5(0)$ & $15(0)$ & $0,18(0)$ & 7,11 & 7,24 & 1,97 & 2,17 \\
\hline 10 & $2,25(0)$ & $2,5(0)$ & $15(0)$ & $0,18(0)$ & 7,02 & 7,21 & 1,97 & 2,06 \\
\hline 11 & $2,25(0)$ & $2,5(0)$ & $15(0)$ & $0,18(0)$ & 7,09 & 7,16 & 1,99 & 2,36 \\
\hline
\end{tabular}
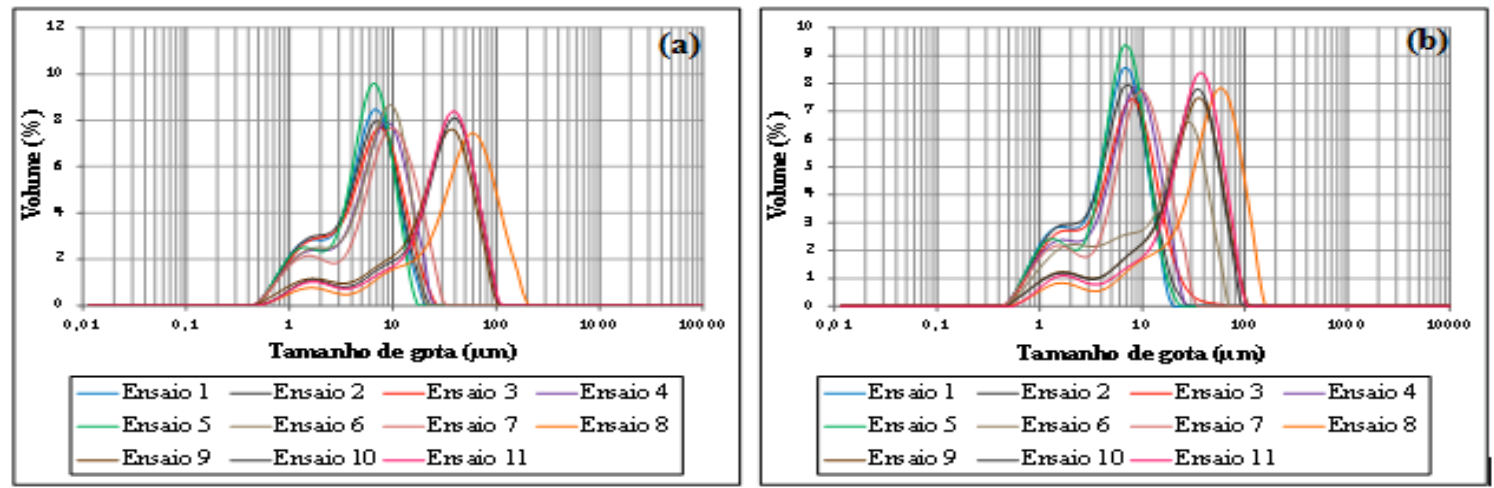

Figura 2: Comportamento das emulsões produzidas com a membrana de 0,2 $\mu \mathrm{m}$, (a) tempo zero e (b) após 60 dias de armazenamento.

\subsection{Emulsões Produzidas com Membrana de $0,8 \mu \mathrm{m}$}

A Figura 3(a) apresenta a distribuição de tamanho de gotas das emulsões obtidas com a membrana de $0,8 \mu \mathrm{m}$, analisadas logo após o preparo. Pode-se observar que as emulsões resultantes apresentam distribuição de tamanho de gotas bimodal, embora a fração de gotas pequenas dos ensaios 


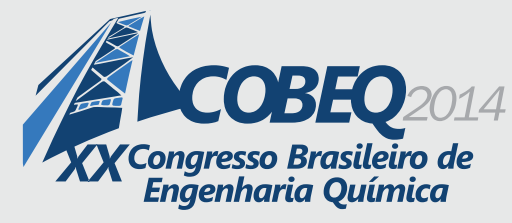

19 a 22 de outubro de 2014
Florianópolis/SC

2, 3, 5 e de 7 a 11 seja menor que $1 \%$ do volume total de gotas, mostrando uma tendência ao comportamento monomodal.

Na Figura 4(b) é nítido o comportamento instável dessas emulsões. A grande diferença entre esses ensaios pode ser explicada devido aos valores dos parâmetros utilizado em cada experimento. Para o ensaio 8, todas as condições de operação utilizadas no processo foram nos menores níveis $(-1)$.
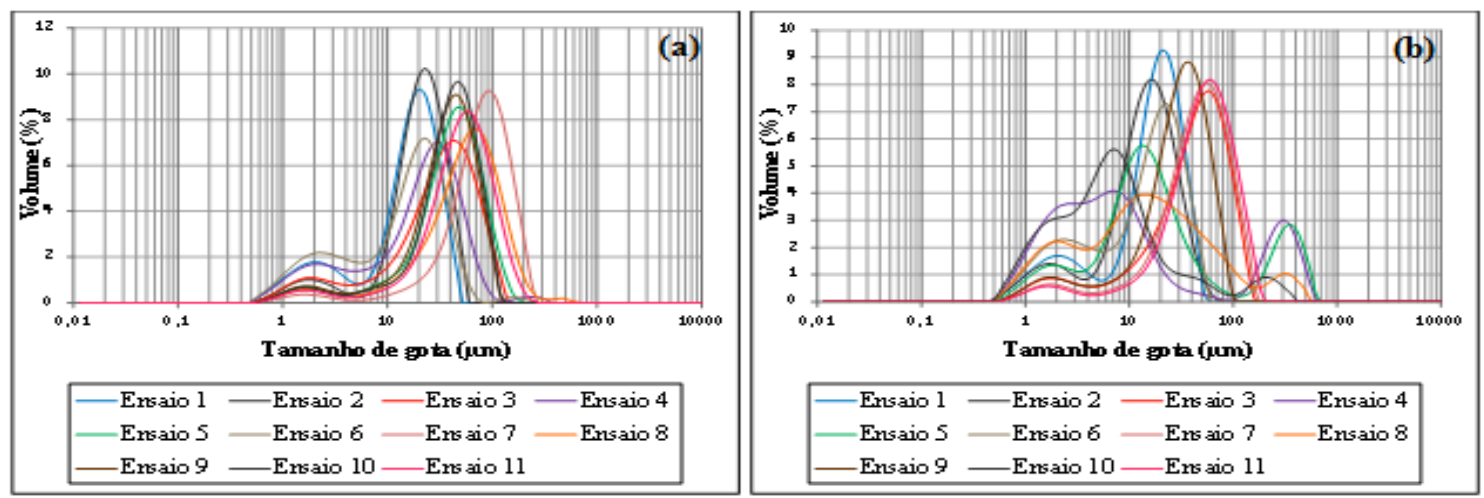

Figura 4: Comportamento das emulsões produzidas com membrana de 0,8 um, (a) tempo zero (b) após 100 dias de armazenamento.

Foram analisadas as respostas em termos do diâmetro médio de gotas $\left(\mathrm{D}_{3,2}\right)$ e dispersão da distribuição de tamanho de gotas (Span) no tempo zero $\left(\mathrm{t}_{0}\right)$ e, após 100 dias de armazenamento $\left(\mathrm{t}_{1}\right)$ para a membrana de $0,8 \mu \mathrm{m}$. Os dados de tamanho médio de gotas e Span para cada condição experimental são apresentados na Tabela 2. No $t_{0}$ os ensaios 7 e 2 apresentam uma estreita distribuição de gotas (menor span), quando comparados aos demais experimentos. Observa que, após 100 dias de armazenamento o comportamento foi bastante diferente do que ocorreu com as emulsões preparadas com a membrana de $0,2 \mu \mathrm{m}$. Enquanto para a membrana de menor tamanho médio de poros o aumento no span com o tempo de armazenamento foi pequeno, para a membrana de $0,8 \mu \mathrm{m}$, em algumas condições (4, 5 e 6), o aumento do span com o tempo de armazenamento foi expressivo, indicando a instabilidade destas emulsões.

Observa-se que os tamanhos médios de gota foram superiores aos obtidos com a membrana de $0,2 \mu \mathrm{m}$. Esse resultado é esperado, uma vez que um tamanho médio de poros menor leva à formação de gotículas menores. No entanto, é interessante notar que apesar do tamanho médio de poros ser 4 vezes maior, o tamanho das gotas aumentou em média 2 vezes. Esse comportamento mostra claramente que o tamanho médio de poros influencia positivamente o tamanho das gotas, mas essa influência é dependente de outros parâmetros, como aqueles investigados no planejamento experimental. Por exemplo, com a membrana de $0,8 \mu \mathrm{m}$ nas condições dos ensaios 6 e 7 foram 
obtidos tamanhos médios de gota 1,2 e 3,9 vezes maiores do que com a membrana de 0,2 $\mu \mathrm{m}$. Ambos ensaios 6 e 7 utilizaram a mesma pressão de operação (1,5 bar para a membrana de $0,2 \mu \mathrm{m}$ e 1,0 bar para a membrana de $0,8 \mu \mathrm{m}$ ). No entanto, o ensaio 6 utilizou mais surfactante, menos óleo e maior velocidade tangencial do que o ensaio 7. Para a membrana de 0,2 essas diferenças nos parâmetros parecem ser desprezíveis, já que o tamanho médio de gotas obtidos são semelhantes $(4,04 \mu \mathrm{m}$ e 3,64 $\mu \mathrm{m}$, respectivamente). Porém, para a membrana de $0,8 \mu \mathrm{m}$, a diferença é bastante elevada $(4,76 \mu \mathrm{m}$ e 14,25 $\mu \mathrm{m}$, respectivamente). Assim, pode-se inferir que o aumento do tamanho de poros da membrana aumenta a importância destes parâmetros frente ao processo de emulsificação.

Tabela 2: Variação de $\mathrm{D}_{32}$ e Span das emulsões obtidas com a membrana de $0,8 \mu \mathrm{m}$ em função da pressão, velocidade tangencial e das concentrações de surfactante e de óleo. $\mathrm{D}_{32}$ apresentado em $\mu \mathrm{m}$. T0 se refere a emulsões analisadas logo após o preparo, e T1 a emulsões analisadas 60 dias após o preparo.

\begin{tabular}{|c|c|c|c|c|c|c|c|c|}
\hline Ensaios & $\begin{array}{c}\text { Pressão } \\
\text { (bar) }\end{array}$ & $\begin{array}{c}\text { Surfactante } \\
(\%) \\
\end{array}$ & $\begin{array}{l}\text { Óleo } \\
(\%)\end{array}$ & $\begin{array}{c}\text { Velocidade } \\
\text { Tangencial }\left(\mathrm{m} \cdot \mathrm{s}^{-1}\right)\end{array}$ & $\begin{array}{c}\mathbf{D}[3,2] \\
\mathbf{T}_{0} \\
\end{array}$ & $\begin{array}{c}\mathbf{D}[3,2] \\
\mathbf{T}_{1} \\
\end{array}$ & $\begin{array}{c}\text { Span } \\
\mathrm{T}_{0}\end{array}$ & $\begin{array}{c}\text { Span } \\
\mathrm{T}_{1} \\
\end{array}$ \\
\hline 1 & $2(+1)$ & $1(-1)$ & $10(-1)$ & $0,24(+1)$ & 5,62 & 5,86 & 1,80 & 1,81 \\
\hline 2 & $2(+1)$ & $4(+1)$ & $10(-1)$ & $0,12(-1)$ & 8,50 & 5,79 & 1,61 & 2,07 \\
\hline 3 & $2(+1)$ & $4(+1)$ & $20(+)$ & $0,12(-1)$ & 8,86 & 11,05 & 2,22 & 2,03 \\
\hline 4 & $1(-1)$ & $4(+1)$ & $20(+)$ & $0,24(+1)$ & 6,01 & 3,69 & 2,27 & 46,45 \\
\hline 5 & $2(+1)$ & $1(-1)$ & $20(+)$ & $0,24(+1)$ & 5,33 & 13,33 & 1,96 & 6,79 \\
\hline 6 & $1(-1)$ & $4(+1)$ & $10(-)$ & $0,24(+1)$ & 4,76 & 12,42 & 2,27 & 3,90 \\
\hline 7 & $1(-1)$ & $1(-1)$ & $20(+)$ & $0,12(-1)$ & 14,25 & 24,22 & 1,59 & 1,88 \\
\hline 8 & $1(-1)$ & $1(-1)$ & $10(-)$ & $0,12(-1)$ & 15,43 & 9,79 & 2,13 & 2,55 \\
\hline 9 & $1,5(0)$ & $2,5(0)$ & $15(0)$ & $0,18(0)$ & 10,23 & 15,72 & 1,74 & 1,81 \\
\hline 10 & $1,5(0)$ & $2,5(0)$ & $15(0)$ & $0,18(0)$ & 13,36 & 15,96 & 1,64 & 1,87 \\
\hline 11 & $1,5(0)$ & $2,5(0)$ & $15(0)$ & $0,18(0)$ & 16,17 & 16,21 & 1,82 & 1,91 \\
\hline
\end{tabular}

A larga distribuição do tamanho das gotas dos ensaios 4 e 5, ocorreu devido à separação de fases, que pode ser visualizada na Figura 4. A instabilidade das emulsões com maior tamanho de gotas é comum, e explicada pelos mecanismos de desestabilização.

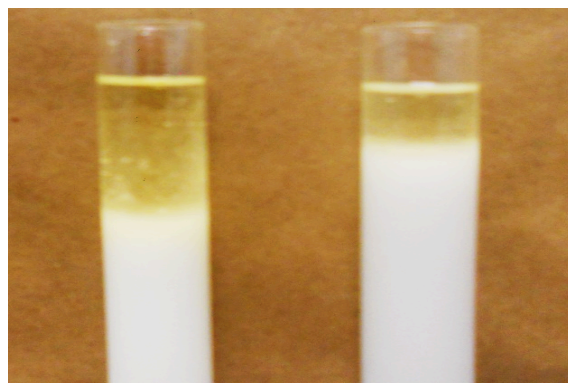

Figura 4: Emulsões obtidas nas condições dos ensaios 4 (esq.) e 5 (dir.) com membrana de 0,8 $\mu$ m. 


\section{CONCLUSÃO}

Membranas cerâmicas microporosas de tamanho médio de poros $0,2 \mu \mathrm{m}$ e $0,8 \mu \mathrm{m}$ foram utilizadas com sucesso para a produção de emulsões de óleo de girassol em água com tamanho médios de gotas variando de $2,9 \mu \mathrm{m}$ a $11,6 \mu \mathrm{m}$, para a membrana de $0,2 \mu \mathrm{m}$; e de 4,8 a $16,2 \mu \mathrm{m}$ para a membrana de $0,8 \mu \mathrm{m}$. A amplitude das distribuições variaram de 1,59 a 2,52 para a membrana de $0,2 \mu \mathrm{m}$, e de 1,59 a 2,27 para a membrana de 0,8 . Quanto à estabilidade das emulsões, pode-se dizer que as emulsões obtidas com a membrana de $0,2 \mu \mathrm{m}$ são bem mais estáveis que aquelas obtidas com a membrana de $0,8 \mu \mathrm{m}$. O presente estudo demonstrou que o tamanho das gotas é dependente do tamanho dos poros da membrana, da pressão, da concentração de óleo, do surfactante e da velocidade tangencial, principalmente para membranas de maior tamanho de poros.

\section{REFERÊNCIAS}

JOSCELYNE, S. M.; GUN, T. Membrane emulsification: a literature review. Journal of Membrane Science, v. 169(1), p. 107-117, 2000.

MATOS, M.; SUÁREZ, M.A.; GUTIÉRREZ, G.; COCA, J.; PAZOS, C. Emulsification with microfiltration ceramic membranes: A different approach to droplet formation mechanism. Journal of Membrane Science, v. 444, p. 345-358, 2013.

NAKASHIMA, T.; SHIMIZU M.; KUKIZAKI, M. Membrane emulsification by microporous glass. Key Eng Mater., v. 61/ 62, p. 513-516, 1991.

NAZIR, K. SCHOREN; BOOM, R. Premix emulsification: A review. Journal of Membrane Science, v. 362(1-2), p. 1-11, 2010.

Agradecimentos: Os autores agradecem à CAPES e CNPq pelo apoio financeiro e ao Laboratório de Farmacotécnica e Cosmetologia (LFC) da UFSC por viabilizar as análises no Mastersizer 2000. 